\title{
Cloudified and Software Defined 5G Networks: Architecture, Solutions, and Emerging Applications
}

\author{
Yin Zhang ${ }^{1} \cdot$ Min Chen ${ }^{2} \cdot$ Roy "Xiaorong" Lai ${ }^{3}$
}

Published online: 30 August 2016

(C) Springer Science+Business Media New York 2016

With the acceleration of human social information accumulation, an effective development in wireless mobile communication is required to deal with ubiquitous connectivity on improving all aspects of people's lifestyles in terms of work, social, and economy. It is predicted that the mobile traffic in 2020 will reach 500 times that in 2010, which imposes new challenges and needs for the development of future mobile communication systems $(5 \mathrm{G})$. $5 \mathrm{G}$ is a multi-service and multi-technology integrated network, meeting the future's needs of dealing with a wide range of big data and the rapid development of numerous businesses, and enhancing users' experiences. $5 \mathrm{G}$ mobile system calls for significant advances in system architecture design and development for the tremendous increase of mobile traffic, the omnipresence of mobile services and the ever-increasing mobile devices.

Fortunately, benefited from both advanced virtualization techniques of cloud computing and software-defined technologies, the integration of Cloud-based Radio Access Network (Cloud RAN), Network Functions Virtualization (NFV), Software-Defined Networking (SDN), Software-Defined Radio (SDR), etc. would further achieve the better spectral efficiency, network capacity, and coverage.

\section{Min Chen \\ minchen@ieee.org \\ Yin Zhang \\ yin.zhang.cn@ieee.org \\ Roy "Xiaorong” Lai \\ roy.lai@ieee.org}

Zhongnan University of Economics and Law, Wuhan, China

2 Huazhong University of Science and Technology, Wuhan, China

3 Confederal Networks Inc., Renton, WA, USA
Motivated by exploring the further potential of cloud computing while integrated with various advanced technologies, this special issue including 6 high quality papers aims to present innovative architectures, solutions, applications and other achievements that will create impact on the design of cloudified and software defined $5 \mathrm{G}$ networks by bringing together academic and industrial researchers to identify and discuss technical challenges related to cloud computing and $5 \mathrm{G}$.

This issue opens with a paper on the security issues of Software Defined Mobile Networks (SDMN), namely "Software-Defined Mobile Networks Security". The authors introduced the structure and key technologies of SDMN. The authors explore relevant security threats and their corresponding countermeasures with respect to the data layer, control layer, application layer, and communication protocols. Moreover, a list of security challenges of SDMN were presented that call for significant research efforts.

In the second paper, "Cloud-Assisted Mood Fatigue Detection System", X. Shi, et al. introduce the basic concept of mood fatigue detection and existing solutions as well as some typical solutions, such as mobile sensing and cloud computing technology. Furthermore, the authors propose a cloud-assisted mood fatigue detection system consisting of wearable devices, clouds data center and application and service manager. Based on the proposed scheme, a real system is established, which is composed of the smart clothing, cloud platform and mobile terminal application.

In the paper titled "Audio-Visual Emotion Recognition Using Big Data towards 5G", M. S. Hossain et al. propose an infrastructure that combines the potential of emotion-aware big data and cloud technology towards $5 \mathrm{G}$, in order to recognize a user's sentiments (e.g., emotion) and behavioral patterns to provide a high quality mobile user experience. Furthermore, a bimodal system of big data emotion recognition is proposed based on the proposed infrastructure. 
Through adequate experiments, it evaluates that the proposed approach achieves $83.10 \%$ emotion recognition accuracy using bimodal inputs.

The fourth paper "Security in Software-Defined Networking: Threats and Countermeasures", co-authored by Z. Shu et al., focuses on the security aspects of SDN, and discusses the characteristics and standards of SDN. Specifically, the security features, the security threats and countermeasures are investigated in detail from three aspects, based on which part of the SDN paradigm they target, i.e., the data forwarding layer, the control layer and the application layer.

In the fifth paper "Efficient Computation Offloading Decision in Mobile Cloud Computing over 5G Network", M. E Khoda et al. propose an intelligent computation offloading system that takes tradeoff decisions for code offloading from a mobile device to cloud server over the $5 \mathrm{G}$ network. In particular, the authors develop a metric for tradeoff decision making that can maximize energy saving while maintain strict latency requirements of user applications in the $5 \mathrm{G}$ system. The experimental results evaluates the proposed system outperforms the state-of-the-art methods in terms of accuracy, computation and energy saving.

In the six paper "Adaptive VM Management with Two Phase Power Consumption Cost Models in Cloud Datacenter", D. Kang et al. propose a two phase based adap- tive resource management (TP-ARM) scheme that migrates VM instances from under-utilized servers that are supposed to be turned off to sustainable ones based on their monitored resource utilizations in real time. In addition, a SelfAdjusting Workload Prediction (SAWP) method is designed to improve the forecasting accuracy of resource utilization even under irregular demand patterns.

In the last paper "Efficient Resource Management for Cloud-enabled Video Surveillance over Next Generation Network", M. A. Hossain et al. propose a computation model to efficiently manage cloud resources for surveillance tasks allocation. The proposed model works on optimizing the trade-off between average service waiting time and longterm service cost, and shows that long-term service cost is inversely proportional to high and balanced utilization of cloud resources.

In the end, we would like to thank all the authors who submitted their research work to this special issue. We also appreciate the contribution of many experts in the field who have participated in the review process and provided constructive suggestions to the authors to improve the contents and presentations of the papers. We would in particular like to thank Professor Imrich Chlamtac, the Editor-in-Chief, for his support and helpful suggestions during the very delicate stages of concluding the special issue. 